\title{
Monitoring Sound Levels and Soundscape Quality in the Living Rooms of Nursing Homes: A Case Study in Flanders (Belgium)
}

\author{
Francesco Aletta ${ }^{1, *} \mathbb{C}^{\mathbb{D}}$, Dick Botteldooren ${ }^{1}$, Pieter Thomas ${ }^{1}{ }^{\mathbb{D}}$, Tara Vander Mynsbrugge ${ }^{2}$, \\ Patricia De Vriendt ${ }^{2,3,4}$, Dominique Van de Velde ${ }^{2,3}$ and Paul Devos ${ }^{1}$ \\ 1 Department of Information Technology, Ghent University, 9052 Ghent, Belgium; \\ dick.botteldooren@ugent.be (D.B.); pieter.thomas@ugent.be (P.T.); p.devos@ugent.be (P.D.) \\ 2 Department of Occupational Therapy, Artevelde University College, 9000 Ghent, Belgium; \\ tara.vandermynsbrugge@arteveldehs.be (T.V.M.); patricia.devriendt@arteveldehs.be (P.D.V.); \\ dominique.vandevelde@arteveldehs.be (D.V.d.V.) \\ 3 Department of Rehabilitation Sciences and Physiotherapy, Ghent University, 9000 Ghent, Belgium \\ 4 Department Gerontology and Frailty in Ageing, Vrije Universiteit Brussel, 1090 Brussels, Belgium \\ * Correspondence: francesco.aletta@ugent.be; Tel.: +32-926-43323
}

Received: 28 July 2017; Accepted: 24 August 2017; Published: 26 August 2017

\begin{abstract}
Recently there has been an increasing interest in the acoustic environment and its perceptual counterpart (i.e., the soundscape) of care facilities and their potential to affect the experience of residents with dementia. There is evidence that too loud sounds or poor soundscape quality more generally can affect negatively the quality of life of people with dementia and increase agitation. The AcustiCare project aims to use the soundscape approach to enhance the Quality of Life (QoL) of residents and to reduce Behavioral and Psychological Symptoms of Dementia (BPSD), as well as improving the everyday experience of nursing homes for both residents and staff members. In order to gain further insights into the sound environments of such facilities, sound level monitoring and soundscape data collection campaigns were conducted in the living rooms of five nursing homes in Flanders. Results showed that sound levels (dB) and loudness levels (sone) did not vary significantly between days of the week, but they did so between moments of the day and between living rooms. From the perceptual point of view, several soundscape attributes and the perceived prominence of different sound source types varied significantly between the living rooms investigated, and a positive correlation was found between sound levels and the number of persons present in the living rooms. These findings claim for further attention on the potential role of the sound domain in nursing homes, which should promote (and not only permit) better living and working conditions for residents and staff members of nursing homes.
\end{abstract}

Keywords: soundscape; indoor sound quality; nursing homes; dementia care

\section{Introduction}

The design and management of care environments is increasingly attracting practitioners and researchers' interest due to the important societal role these facilities account for. In spite of its environmental significance, sound is often disregarded as an influencing factor of the users' experience in such contexts. Specific standards for the acoustic performance of care facilities are often missing and this is likely to lead, in turn, to noisy and unpleasant sound environments. Particularly, the perception of sound environments is of utmost relevance in places like hospitals, care facilities, or nursing homes, since these places often deal with "vulnerable" users, like older adults or people with intellectual disabilities [1]. 
Soundscape refers to the human perception of an acoustic environment in context [2]. While the concept has attracted an increasing interest in urban studies, and outdoor environments more in general, it also applies to indoor contexts, particularly when they serve "public" functions, like service buildings, public libraries, transportation hubs, restaurants, or other commercial facilities [3-5].

There are relatively few examples of research investigating the soundscape quality of care facilities and, in particular, of nursing homes. There is also a lack of awareness of the importance of the "quality" of sound for the Quality of Life (QoL) in daily care. Van den Bosch et al. [6] have indeed claimed for further research attention on the role of sound and its potential to reduce behavioural problems in such environments to enhance the quality of life of residents [6,7]. They have pointed out that this often depends on "pleasantness" and "safety" rather than "objective" sound levels.

In particular, persons with dementia are vulnerable to environmental disturbance [6,7]. Dementia is an overall term that describes a wide range of symptoms associated with a decline in memory or other cognitive skills, severe enough to reduce a person's ability to perform everyday activities. Another characteristic is the presence of Behavioural and Psychological Symptoms of Dementia (BPSD), often seen in more severe stages of dementia. BPSD are commonly understood as symptoms of disturbed perception, thought content, mood, and behaviour, which frequently occur in people with dementia. Although BPSD are considered as a characteristic of dementia, evidence suggests that environmental factors may trigger BPSD [6].

Given the fact that environmental factors, and specifically acoustics, impact BPSD [1,6,7], the AcustiCare project started in October 2016 with the aim of using the soundscape approach to enhance the everyday experience of nursing homes for both residents with dementia and staff members. For this purpose, five nursing homes in Flanders hosting people with dementia and BPSD were involved in the project. One of the first objectives of the research project was characterising the physical acoustic environment (through "objective" data) and its perception (through "subjective" data) in the living rooms of the nursing homes. The rationale for selecting the living rooms is that they are the key spaces for the functionality of the facility, because they form part of the context where nursing homes' residents spend most of their daily life and undertake daily activities such as eating, drinking coffee, having social talks, playing games, etc. Hence, it seemed reasonable to start from these environments, since "aging in place" is considered as an important precursor for quality of life provided that the environment offers safety, a feeling of being home, and a sense of place and time $[8,9]$.

\section{Objectives of the Study}

The approach of this study was twofold: objective and subjective. On the one hand it aimed at getting an overall impression of the "acoustic climate" of the living rooms included in the project; on the other hand it aimed at exploring, in the most systematic possible way, the soundscape quality of the living rooms.

In particular, for the former aspect, the specific goals were testing if there are significant differences in terms of sound levels and loudness: (a) between different days of the week; (b) between different living rooms; and (c) between different time slots of the day. The research questions (a)-(c) are addressed together in Sections 2.1, 3.1 and 4.1 (Methods, Results and Discussion, accordingly). For the latter aspect, the specific goals were testing if there are significant differences in terms of soundscape quality and the prominence of sound sources type: (d) between different time slots of the day; and (e) between different living rooms. Research questions (d) and (e) are covered by Sections 2.2, 3.2 and 4.2. Furthermore, this study also analysed the associations between the sound levels recorded during the monitoring period and the number of persons present in the living rooms in different moments of the day, to investigate $(\mathrm{f})$ what is the potential contribution of such (anthropic) sources to the overall sound environment. The research question (f) was addressed in Section 2.2, Sections 3.3 and 4.3. 
For all the above mentioned goals, sound level monitoring and a soundscape data collection campaign were organized in each facility using a low-cost sensors' network and a revised version of a soundscape protocol available in the literature, accordingly.

\section{Methods}

This section describes the case studies where both the sound level monitoring and perceptual data collection about the soundscape assessment were performed. Overall, the sound levels were monitored in nine living rooms (in five nursing homes), while only five living rooms (one in each nursing home) were considered for the soundscape assessment. Every living room typically serves 15-25 persons (depending on the size). While the sound level monitoring was performed continuously during almost a whole week, the collection of perceptual data only covered single days during day time. For the sake of confidentiality, the names of the living rooms are coded as: VH1, VH2, SJ, LH1, LH2, SV1, SV2, SP1, and SP2. Same-code living rooms belong to the same nursing home. The research was approved by the Ethical Committee that reviewed the study, registered under the number B670 $201630512-20161501$ at the commission for Medical Ethics of the Faculty of Medicine and Health Sciences at Ghent University and Ghent University Hospital.

\subsection{Sound Level Monitoring of the Living Rooms}

One of the main aims of this work was gathering information about the overall acoustic environment of the living rooms of nursing homes. For this purpose, cost-effective sensor nodes were installed in the nine living rooms. The nodes were installed far from specific noise sources which could exaggerate the sound levels, but close enough to where most activities took place in such spaces. Figure 1 shows an example of installation in one of the living rooms.


Figure 1. An example of installation of a node in one of the living rooms of the study. The right figure shows the overall context, the left figure shows a close-up of the red box in the right figure.

The nodes continuously measured 1/3-octave band levels, with a temporal resolution of $125 \mathrm{ms,}$ from 07:00 a.m. Monday to 07:00 a.m. Friday, during a typical week of activity of the nursing homes, between December 2016 and February 2017. During the observation period, data were sent over the internet to the Ghent University server infrastructure located in Ghent, Belgium. Data were subsequently further processed through an agent-based approach and stored in a warehouse database. The A-weighted sound equivalent levels were then calculated on a 15-min basis ( $\left.\mathrm{L}_{\text {Aeq-15min }}\right)$ for all data available, for each sensor node. Figure 2 shows a general overview of the system, and more details on the architecture of the sensor network can be retrieved in refs. [10,11]. Figure 3 shows the daily patterns of the sound equivalent levels in the living rooms during the corresponding monitoring week. 


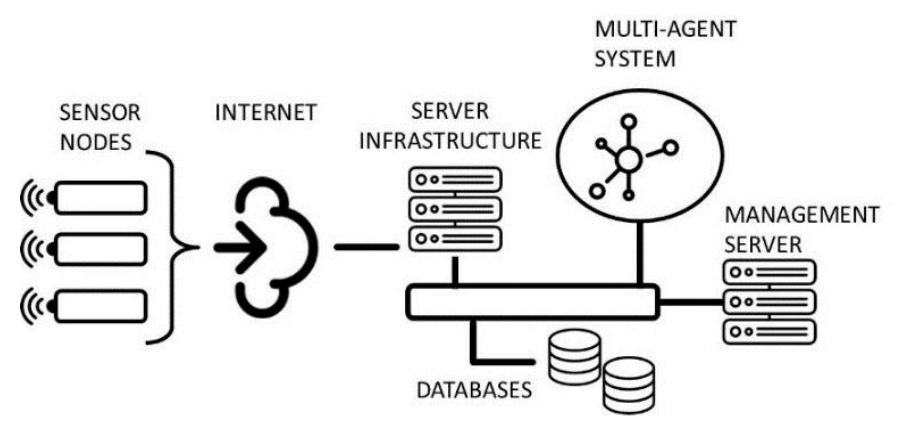

Figure 2. General overview of the sound monitoring sensor network architecture.

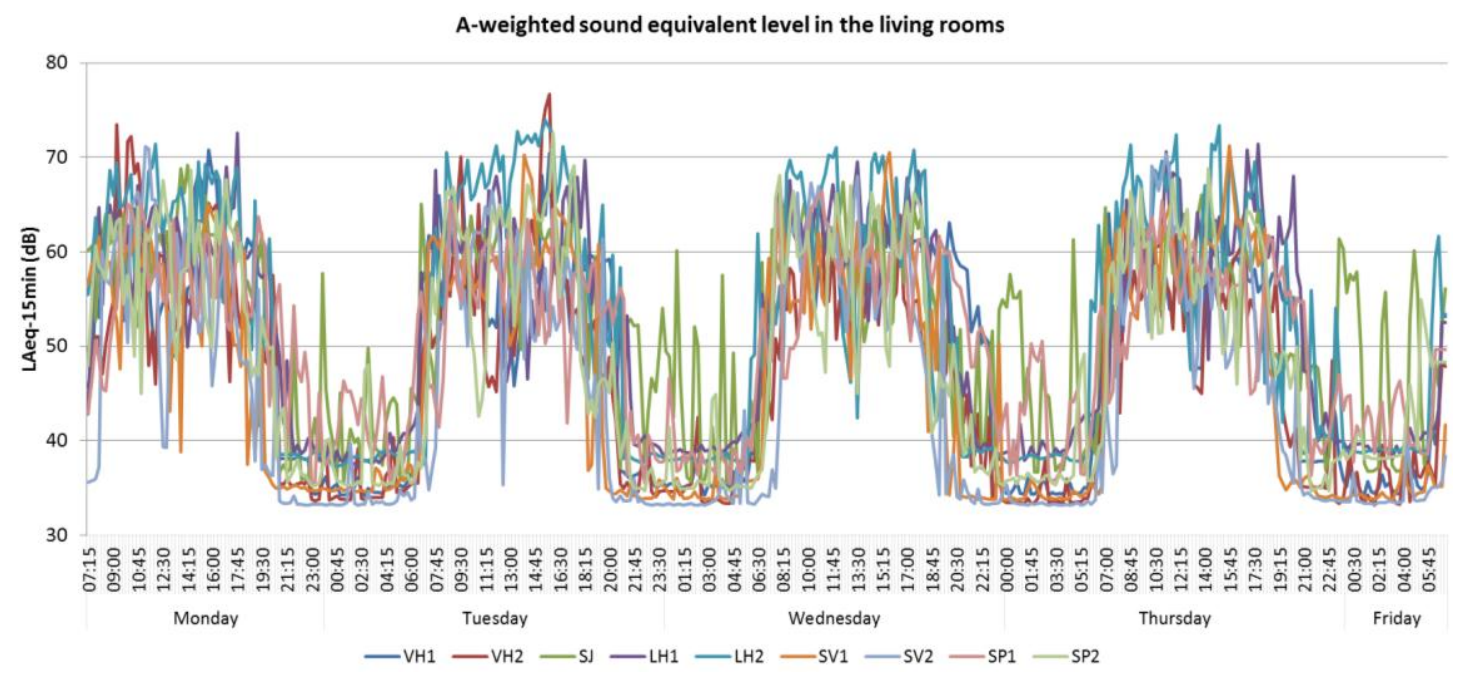

Figure 3. Equivalent sound level patterns in the living rooms during the monitoring week.

To gain further insights into the acoustical characteristics of the living rooms, volumetric data and reverberation times were measured; these are reported in Table 1. The reverberation time (T20) was measured in accordance with the standard ISO 3382-2:2008 (Acoustics-Measurement of room acoustic parameters-Part 2: Reverberation time in ordinary rooms). It was decided to opt for T20 instead of T30 for practical reasons. Due to the potentially sensitive receivers in the proximity of where the measurements were being performed, it was not suitable to use sounds that were too loud. So, to avoid potentially poor signal-to-noise ratio values, it was decided to extrapolate the reverberation time from the $20-\mathrm{dB}$ decay.

Table 1. Volumetric data and reverberation time for the nine investigated living rooms.

\begin{tabular}{ccc}
\hline Living Room & $\approx$ Volume $\left(\mathbf{m}^{\mathbf{3}}\right)$ & T20 $^{\mathbf{1}} \mathbf{( s )}$ \\
\hline VH1 & 615 & 0.91 \\
VH2 & 217 & 1.11 \\
SJ & 264 & 0.84 \\
LH1 & 206 & 0.80 \\
LH2 & 222 & 1.30 \\
SV1 & 124 & 0.36 \\
SV2 & 121 & 0.40 \\
SP1 & 342 & 1.58 \\
SP2 & 499 & 1.23
\end{tabular}

${ }^{1}$ The T20 reported in the table is an arithmetic average of the T20 values in the $500 \mathrm{~Hz}-2 \mathrm{kHz}$ frequency range. 


\subsection{Soundscape Quality Assessment of the Living Rooms}

There is still no clear agreement on what is the best method to collect soundscape data and thus characterize and/or assess in a standard way soundscape qualities [12]. The International Organization for Standardization (ISO) set a working group that is currently developing Part 2 of the norm mentioned in reference [2] (ISO/DIS 12913-2 Acoustics-Soundscape-Part 2: Data collection and reporting requirements), which should overcome this issue, but no consensus has been reached so far. Nevertheless, in the literature, a number of protocols have been proved to perform quite consistently in representing different soundscape dimensions [13-16], and some of them have also been used specifically in care facilities [1]. The questionnaire used to gather soundscape data was indeed a revised version adapted from some of these protocols.

Data were collected using an online form accessed through a tablet provided to a researcher. Table 2 reports the questions used for the soundscape assessment, where for each item, the researcher could express a score on an ordinal scale ranging from 0 to 10 . The same procedure and assessment protocol were implemented in all of the observed living rooms. The researcher covered a 12-h period of observation, from 07:00 a.m. to 07:00 p.m. Data collection took place on Thursdays, from December 2016 to February 2017, one day in each facility (i.e., $60 \mathrm{~h}$ of observation overall). The researcher was sitting in the living room, avoiding interaction with staff, residents, and their family members and/or friends, when present. Every $30 \mathrm{~min}$, the researcher completed the questionnaire described in Table 2, considering the soundscape overall across the last 30-min slot (this task was supported by a vibration-only alarm set to warn the researcher every $30 \mathrm{~min}$ ).

Table 2. Questionnaire used during the soundscape observation in the nursing homes.

\begin{tabular}{|c|c|c|}
\hline ID & Question & Extremes of the Scales [0-10] \\
\hline Q1 & $\begin{array}{l}\text { "Overall, how would you describe the present surrounding sound } \\
\text { environment?" }\end{array}$ & Very bad-Very good \\
\hline Q2 & $\begin{array}{l}\text { "Overall, to what extent is the present surrounding sound environment } \\
\text { appropriate to the present place?" }\end{array}$ & Not at all-Perfectly \\
\hline Q3 & $\begin{array}{l}\text { "To what extent do you presently hear the following seven types of } \\
\text { sounds?" (Installation sounds-e.g., fan/ventilation noise, medical } \\
\text { equipment, telephone...; Operational sounds-e.g., door slamming, } \\
\text { trolleys passing-by, kitchen functions...; Electronic sounds-e.g., TV, } \\
\text { radio, reproduced music, toys...; Environmental noise-e.g., } \\
\text { transportation noise, construction noise, birdsong, wind, rain, sounds } \\
\text { from people outside...; Human sounds-VOCAL—e.g., voices, laughter, } \\
\text { sounds from individuals in the room...; Human } \\
\text { sounds-NON-VOCAL—e.g., footsteps, clapping hands, hitting } \\
\text { objects...; Pets sounds-e.g., birds in a cage, cats, dogs...) }\end{array}$ & $\begin{array}{l}\text { Do not hear at all-Dominates } \\
\text { completely }\end{array}$ \\
\hline Q4 & $\begin{array}{l}\text { "For each of the ten scales below, to what extent do you agree or } \\
\text { disagree that the present surrounding sound environment is ... } \\
\text { (pleasant; chaotic; vibrant; uneventful; calm; annoying; eventful; } \\
\text { monotonous; safe; intimate)?" }\end{array}$ & Not at all-Completely \\
\hline
\end{tabular}

At the same time, the researcher took note of the number of persons present in the living room. The temporal evolution of the overall presence of persons in the living room (aggregating staff, residents, and family members or friends) as a function of the time slots of the observation period in the different living rooms is reported in Figure 4. Data represent "overall" presence (e.g., disregarding people leaving/entering the living room for short periods) during the previous 30-min slot (i.e., data point "10:00" represents presence between 09:30 and 10:00 a.m., and so on). This was assessed by the researcher. 




Figure 4. Overall presence in the living rooms during the observation day.

\section{Results}

Within the framework of this paper, acoustic metrics (i.e., the physical dataset) are meant to inform and support the analysis of soundscape quality (i.e., the perceptual dataset). Therefore, it was decided to consider only the sound level monitoring during the time slots of the day that were covered by the soundscape assessment protocol. Thus, sound levels were analyzed for four days (from Mondays to Thursdays, since the monitoring was interrupted on Friday mornings), considering the interval from 07:00 a.m. to 07:00 p.m. Furthermore, in order to get more insights into the physical acoustic environments of the investigated living rooms, loudness was computed starting from the original signals as additional psychoacoustic parameter [17].

This section is divided in three parts: Section 3.1 deals with statistical differences of sound levels and loudness with respect to temporal factors and between living rooms. Section 3.2 approaches the same differences from the soundscape (i.e., perceptual) data angle. Finally, Section 3.3 considers the associations between equivalent sound levels and the number of people in the living rooms.

\subsection{Sound Level Monitoring of the Living Rooms}

Using the data from the sensor nodes, statistical tests were performed to investigate potential differences in the A-weighted equivalent sound levels ( $\mathrm{dB}$ ) and loudness levels (sone), with respect to three main factors, namely: (1) the day of the week; (2) the 15-min time slot of the monitoring period; and (3) the living room factor itself. Three analyses were run separately. Since data failed to meet the normality assumption, an alternative non-parametric test was opted for: the Kruskal-Wallis statistics test.

To address the research question (a), the first two tests were performed. A first Kruskal-Wallis $\mathrm{H}$ test was performed to determine if there were differences in $\mathrm{L}_{\text {Aeq-15min }}$ scores between the four days: the "Monday", "Tuesday", "Wednesday", and "Thursday" groups. Distributions of $\mathrm{L}_{\text {Aeq- }}-15 \mathrm{~min}$ scores were not similar for all groups, as assessed by visual inspection of a boxplot reported in Figure 5. The mean rank of $\mathrm{L}_{\text {Aeq-15min }}$ scores was not statistically significantly different between groups, $\chi^{2}(3)=3.478, p=0.324$. Likewise, another Kruskal-Wallis $\mathrm{H}$ test was performed to determine if there were differences in Loudness-15min scores. Distributions of Loudness-15min scores were not similar for all groups, as assessed by visual inspection of a boxplot reported in Figure 6 . The mean rank of $\mathrm{L}_{\text {Aeq-15min }}$ scores was not statistically significantly different between groups, $\chi^{2}(3)=2.583, p=0.460$. 


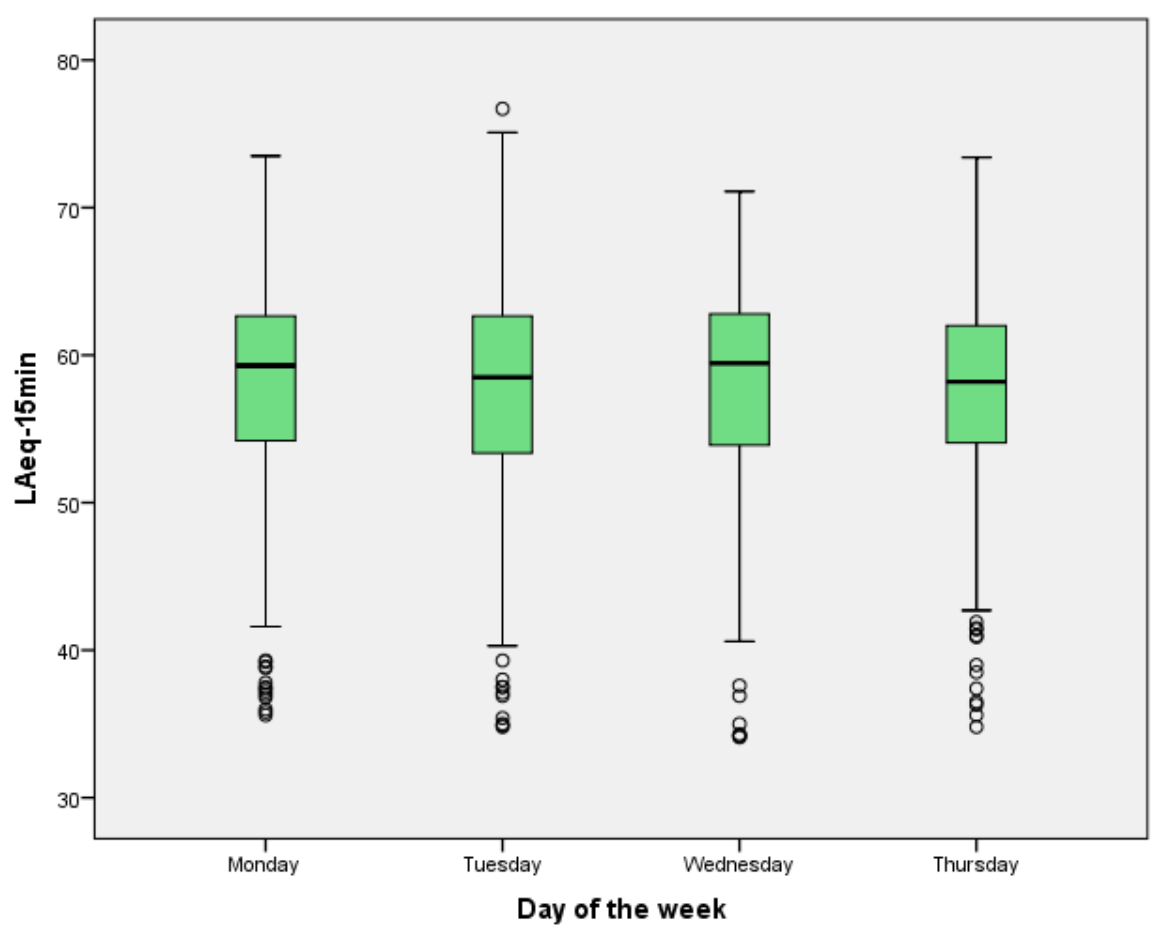

Figure 5. Distributions of $\mathrm{L}_{\text {Aeq-15min }}$ scores as a function of the day of the week.

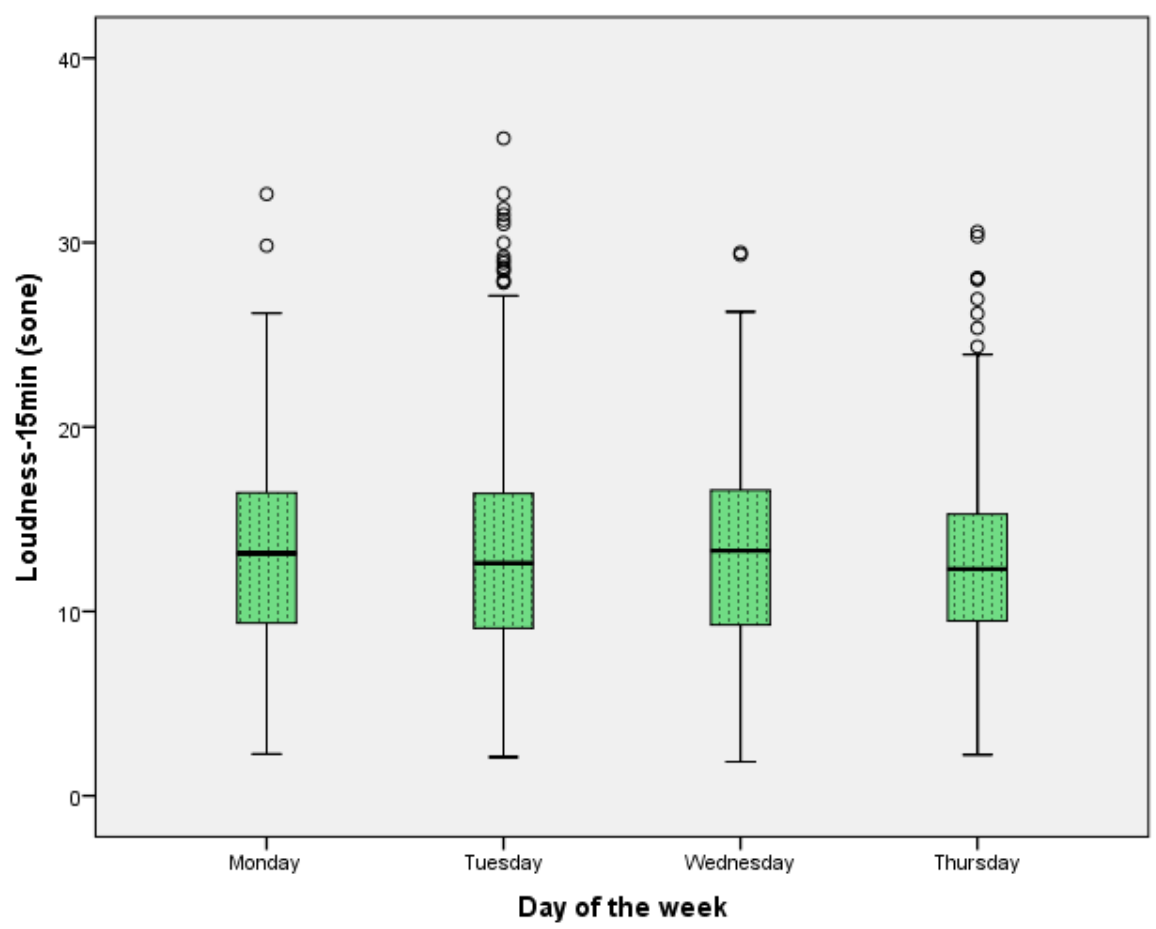

Figure 6. Distributions of Loudness-15min scores as a function of the day of the week.

The lack of statistically significant differences between different days of the week was not surprising since during preliminary meetings between the research group and the management teams of the nursing homes it had been reported that activities in the living rooms tend to follow always the same schedule, to provide residents with regular daily patterns. This is, thus, also reflected in the occurring sound sources. A non-systematic visual inspection of the spectrograms of the sound 
level recordings, compared with some field notes collected on site, confirmed this general circumstance. For instance, Figure 7 represents the five-minute interval between 12:00 and 12:05 p.m. in the living room VH1 during the four full days of noise monitoring (Monday to Thursday). It can be noted that some sound sources are repeated over the week, emerging from a similar background noise.
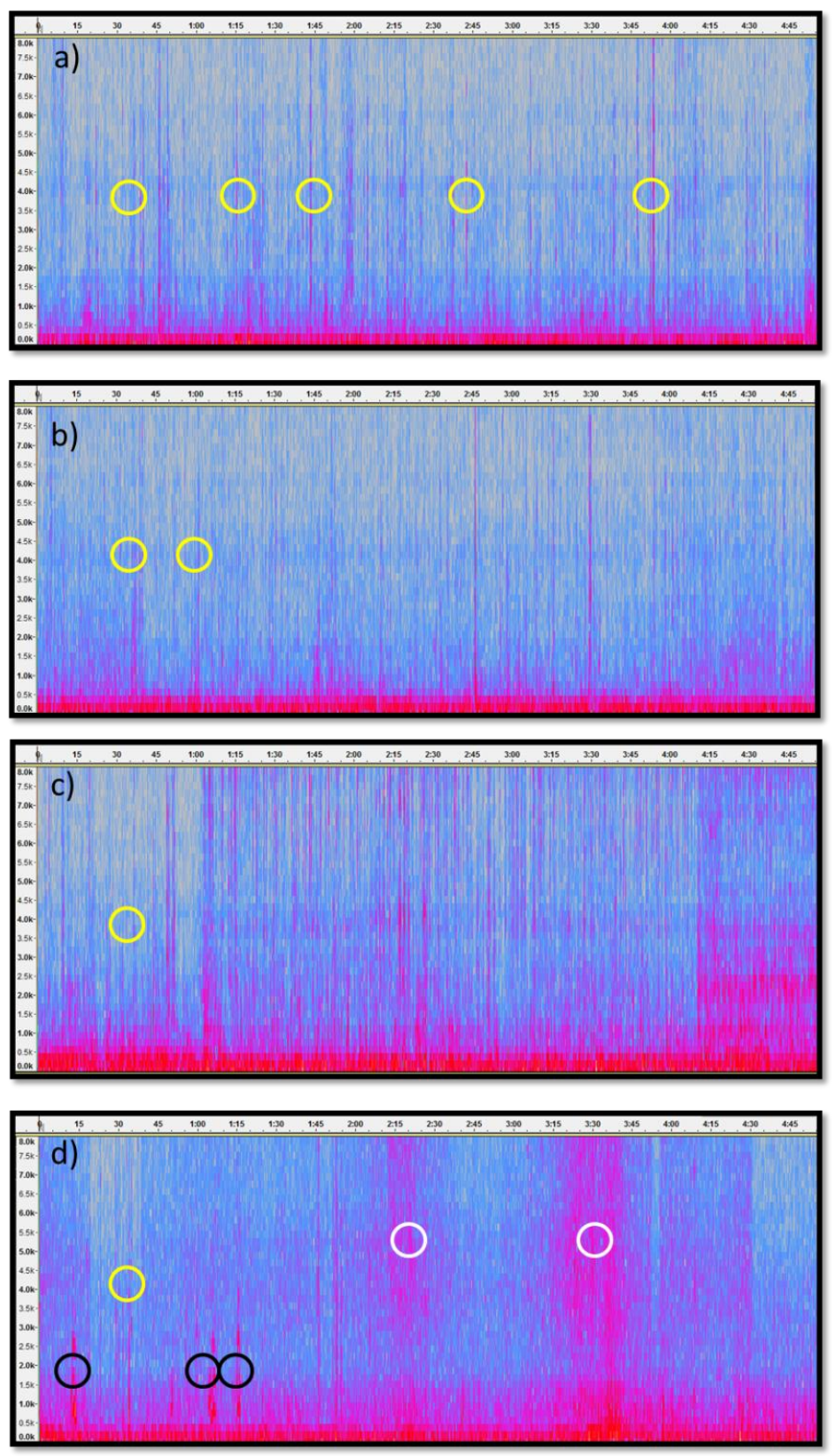

Figure 7. Example of time-frequency spectrograms of the sound levels in VH1: (a) Monday; (b) Tuesday; (c) Wednesday; (d) Thursday. Yellow circles identify examples of sounds of cutlery and kitchenware while lunch was being served to residents by staff members. White circles identify a cleaning machine; black circles identify a vocalizing, agitated resident during lunch.

Subsequently, in order to address the research question (b), a Kruskal-Wallis $\mathrm{H}$ test was performed to determine if there were differences in $\mathrm{L}_{\mathrm{Aeq}-15 \min }$ scores between the nine living rooms: the "VH1", "VH2", "SJ", "LH1", "LH2", "SV1", "SV2", "SP1", and "SP2" groups. Distributions of $\mathrm{L}_{\mathrm{Aeq}-15 \mathrm{~min}}$ scores were not similar for all groups, as assessed by visual inspection of a boxplot reported in Figure 8 . The mean ranks of $\mathrm{L}_{\text {Aeq-15min }}$ scores were statistically significantly different between groups, 
$\chi^{2}(8)=224.711, p<0.001$. Likewise, the distributions for the Loudness $-15 \mathrm{~min}$ scores were not similar for all groups, as assessed by visual inspection of a boxplot reported in Figure 9. The mean ranks of Loudness $-15 \mathrm{~min}$ scores were statistically significantly different between groups, $\chi^{2}(8)=309.929$, $p<0.001$.

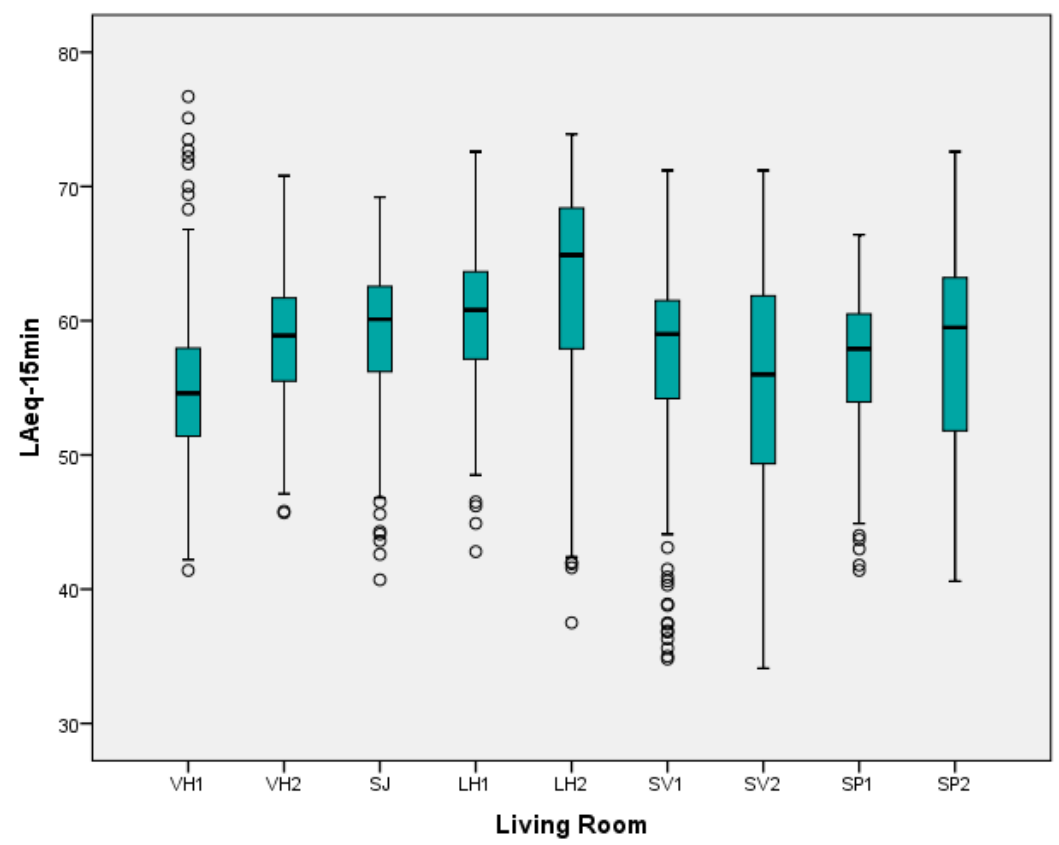

Figure 8. Distributions of $\mathrm{L}_{\mathrm{Aeq}-15 \mathrm{~min}}$ scores as a function of the living rooms.

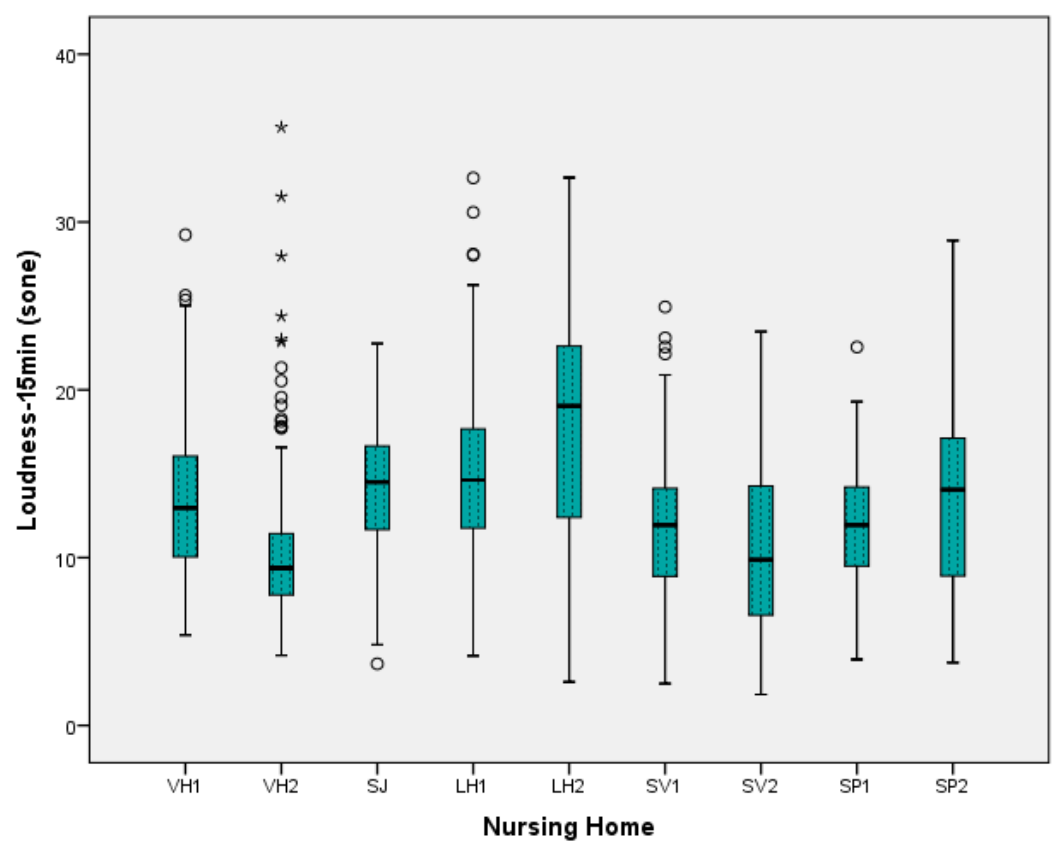

Figure 9. Distributions of Loudness-15min scores as a function of the living rooms.

Eventually, to address research question (c), another Kruskal-Wallis $\mathrm{H}$ test was performed to determine if there were differences in $\mathrm{L}_{\mathrm{Aeq}-15 \mathrm{~min}}$ scores between the different time slots of the monitoring periods: the groups from “07:00" to "19:00". Distributions of $\mathrm{L}_{\text {Aeq-15min }}$ scores were not similar for all groups, as assessed by visual inspection of a boxplot reported in Figure 10. The mean 
ranks of $\mathrm{L}_{\text {Aeq-15min }}$ scores were statistically significantly different between groups, $\chi^{2}(12)=312.837$,

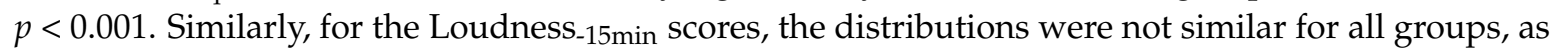
assessed by visual inspection of a boxplot reported in Figure 11. The mean ranks of Loudness-15min scores were statistically significantly different between groups, $\chi^{2}(12)=232.486, p<0.001$.



Figure 10. Distributions of $\mathrm{L}_{\mathrm{A} \text { eq-15min }}$ scores as a function of the time slot.



Figure 11. Distributions of Loudness-15min scores as a function of the time slot.

Comparing the results of the equivalent sound levels and loudness levels analyses, one can see that the latter reflects the former, at least in terms of statistical difference between various experimental conditions. The exponential relationship between $\mathrm{L}_{\mathrm{Aeq}}-15 \mathrm{~min}$ and Loudness $-15 \mathrm{~min}$ scores across the 
whole dataset is depicted in Figure 12. This is in line with previous literature on the association between acoustic and psychoacoustic parameters [17]. Figure 12 indeed shows a good match between the exponential trend of recorded data and the conversion between loudness in decibels and sones proposed by Fastl and Zwicker [17].

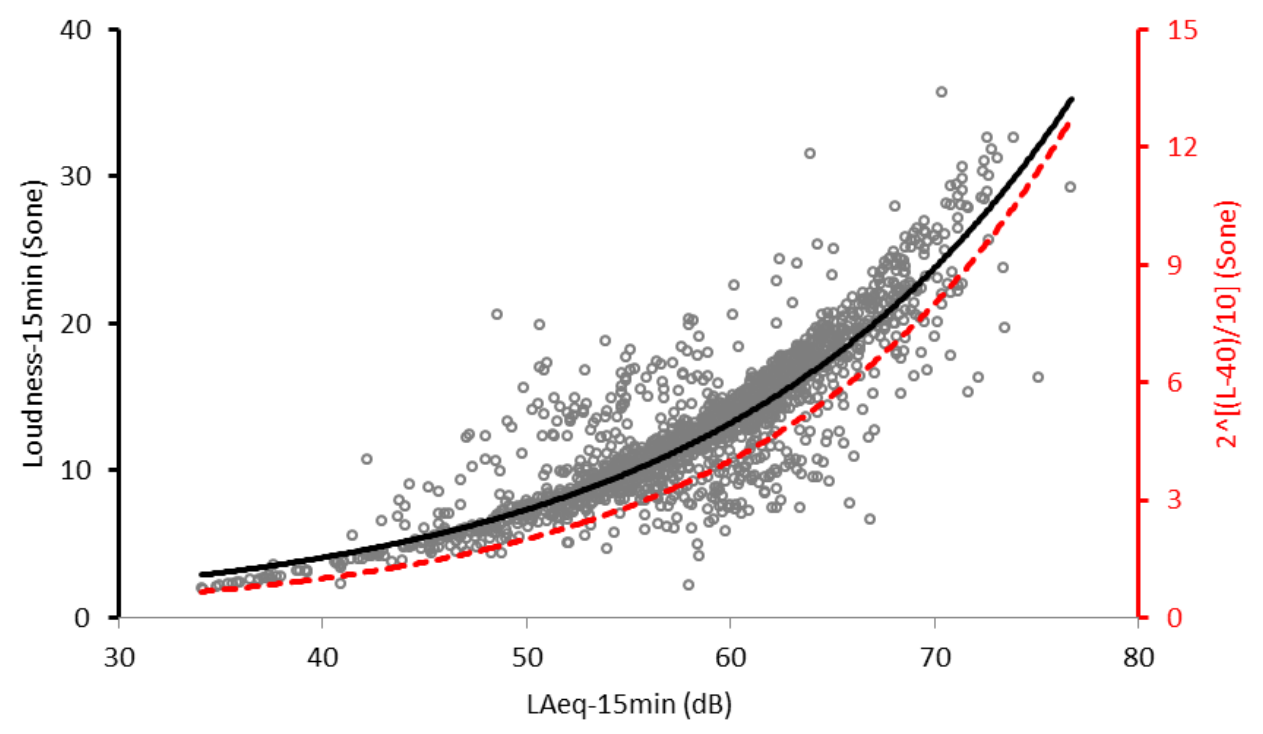

Figure 12. Scatter-plot of the $\mathrm{L}_{\mathrm{A} \text { eq-15min }}$ scores vs. the Loudness-15min scores (black line), compared with the theoretical formulation by Fastl and Zwicker [17] (red dashed line).

\subsection{Soundscape Quality Assessment the Living Rooms}

For the perceptual data, a similar statistical approach with a non-parametric test was used, as for the equivalent sound levels and loudness, since data failed to meet the normality assumption. Soundscape assessments referred to a different time resolution of the observation sessions (i.e., 30-min slots from 07:00 a.m. to 07:00 p.m.), and five living rooms were covered, namely, VH1, SJ, LH1, SV1, and SP1. This single-day approach was also justified by the lack of difference experienced between different days in terms of objective parameters, as previously shown in Section 3.1.

Firstly, in order to address research question (d), the Kruskal-Wallis $\mathrm{H}$ test was performed to determine if there were differences in overall soundscape quality scores (Q1, in Table 2), overall soundscape appropriateness scores $(\mathrm{Q} 2$, in Table 2), prominence of sound source types scores (each of the seven items of Q3, in Table 2) and soundscape attributes scores (each of the ten items of Q4, in Table 2) between the different time slots of the observation sessions: the groups from "07:00" to "19:00". Distributions of all the variables' scores were not similar for all groups, as assessed by visual inspection of a boxplot. The mean rank of all variables' scores was not statistically significantly different between groups ( $p>0.05$ for all tested variables).

Another set of Kruskal-Wallis $\mathrm{H}$ tests was performed to address research question (e) and to assess the differences between the same variables with respect to the living rooms grouping factor. The distributions of all the variables' scores were not similar for all groups, as assessed by visual inspection of the boxplots reported in Figures 13-15. The mean ranks of all the variables' scores were statistically significantly different between groups, as reported in Table 3. In particular, pairwise comparisons were performed for Q1 and Q2 using Dunn's procedure [18] with a Bonferroni correction for multiple comparisons. Adjusted $p$-values are presented. This post hoc analysis for Q1 revealed statistically significant differences in overall soundscape quality scores between: the SP1 (mean rank $=23.18$ ) and SJ (mean rank = 71.00) groups $(p<0.001)$; the SP1 and SV1 (mean rank $=70.93$ ) groups $(p<0.001)$; and the SP1 and VH1 (mean rank $=60.50)$ groups $(p=0.004)$. Furthermore, the post hoc analysis also revealed statistically significant differences in overall soundscape quality scores 
between: the LH1 (mean rank $=41.83$ ) and SJ groups $(p=0.009)$; and the LH1 and the SV1 groups $(p=0.009)$. Likewise, the post hoc analysis for Q2 revealed statistically significant differences in overall soundscape appropriateness scores between: the SP1 (mean rank $=25.77$ ) and SV1 (mean rank $=67.11$ ) groups $(p<0.001)$; and the SP1 and SJ (mean rank $=78.09)$ groups $(p<0.001)$. Furthermore, the post hoc analysis also revealed statistically significant differences in overall soundscape appropriateness scores between: the LH1 (mean rank $=39.29)$ and SV1 groups $(p=0.016)$; and the LH1 and the SJ groups $(p<0.001)$.



Figure 13. Distributions of Q1 and Q2 scores as a function of the living rooms.

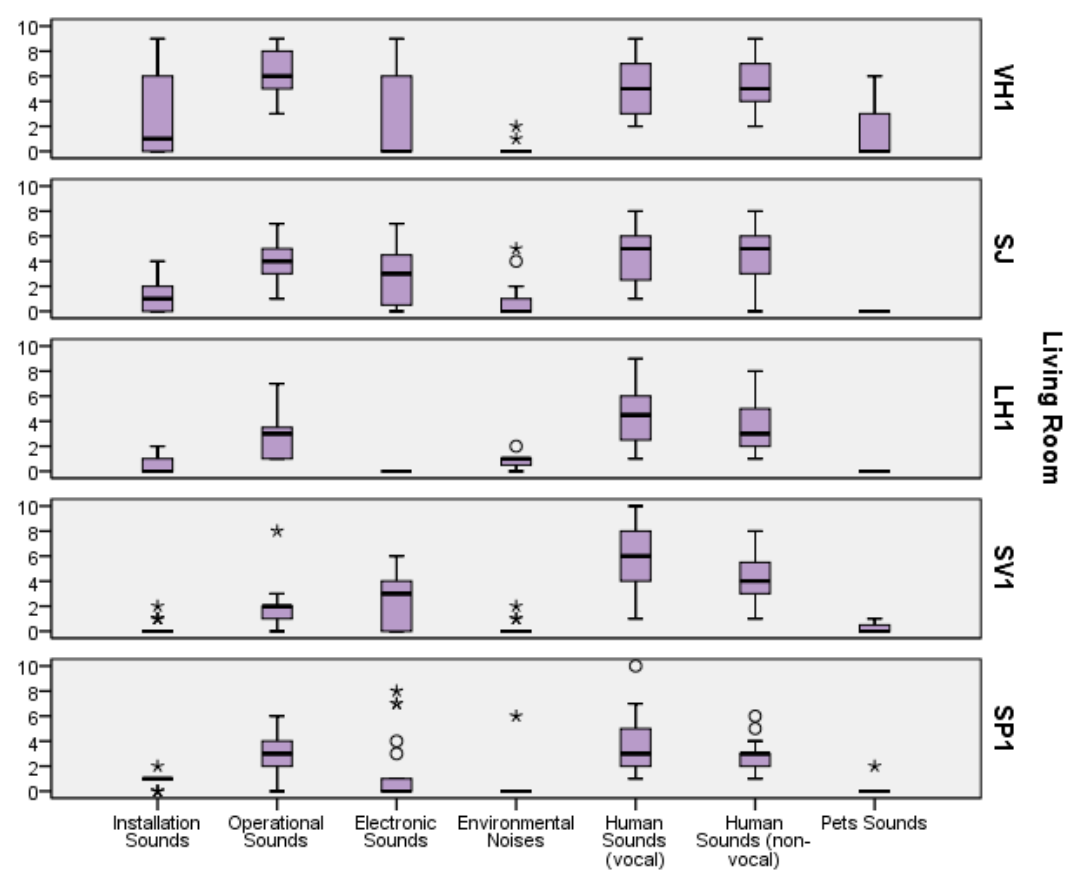

Figure 14. Distributions of Q3 scores for its seven items, sorted by living room. 



Figure 15. Distributions of Q4 scores for its ten items, sorted by living room.

Table 3. Test statistic values and asymptotic significance (2-sided test).

\begin{tabular}{|c|c|c|c|}
\hline ID & Question/Item & Chi-Square $\left(\chi^{2}\right)^{1}$ & $p$-Value \\
\hline Q1 & $\begin{array}{l}\text { "Overall, how would you describe the present } \\
\text { surrounding sound environment?" }\end{array}$ & 42.106 & $p<0.001$ \\
\hline Q2 & $\begin{array}{l}\text { "Overall to what extent is the present surrounding sound } \\
\text { environment appropriate to the present place?" }\end{array}$ & 43.783 & $p<0.001$ \\
\hline \multirow{7}{*}{ Q3 } & Installation sounds & 23.056 & $p<0.001$ \\
\hline & Operational sounds & 38.326 & $p<0.001$ \\
\hline & Electronic sounds & 27.407 & $p<0.001$ \\
\hline & Environmental noise & 29.611 & $p<0.001$ \\
\hline & Human sounds-VOCAL & 11.590 & $p=0.021$ \\
\hline & Human sounds-NON-VOCAL & 17.063 & $p=0.002$ \\
\hline & Pets sounds & 21.661 & $p<0.001$ \\
\hline \multirow{10}{*}{ Q4 } & Pleasant & 30.055 & $p<0.001$ \\
\hline & Chaotic & 17.992 & $p=0.001$ \\
\hline & Vibrant & 34.586 & $p<0.001$ \\
\hline & Uneventful & 22.989 & $p<0.001$ \\
\hline & Calm & 12.934 & $p=0.012$ \\
\hline & Annoying & 12.571 & $p=0.014$ \\
\hline & Eventful & 27.649 & $p<0.001$ \\
\hline & Monotonous & 20.587 & $p<0.001$ \\
\hline & Safe & 46.518 & $p<0.001$ \\
\hline & Intimate & 39.883 & $p<0.001$ \\
\hline
\end{tabular}

${ }^{1}$ All values refer to $\mathrm{N}=105$ and 4 degrees of freedom.

\subsection{Sound Levels and Presence in the Living Rooms}

In order to investigate a potential relationship between the equivalent sound levels measured with the sensor nodes and the number of persons in the living rooms counted by the researcher during the observation sessions in the living rooms, a Spearman's rank-order correlation was performed, which addressed research question (f). There was a moderately positive correlation between sound levels and persons in the living room, $r_{\mathrm{s}}(123)=0.433, p<0.001$. The analysis showed that the linear 
regression trend is positive, as assessed by visual inspection of a scatterplot reported in Figure 16, when considering the living rooms separately.

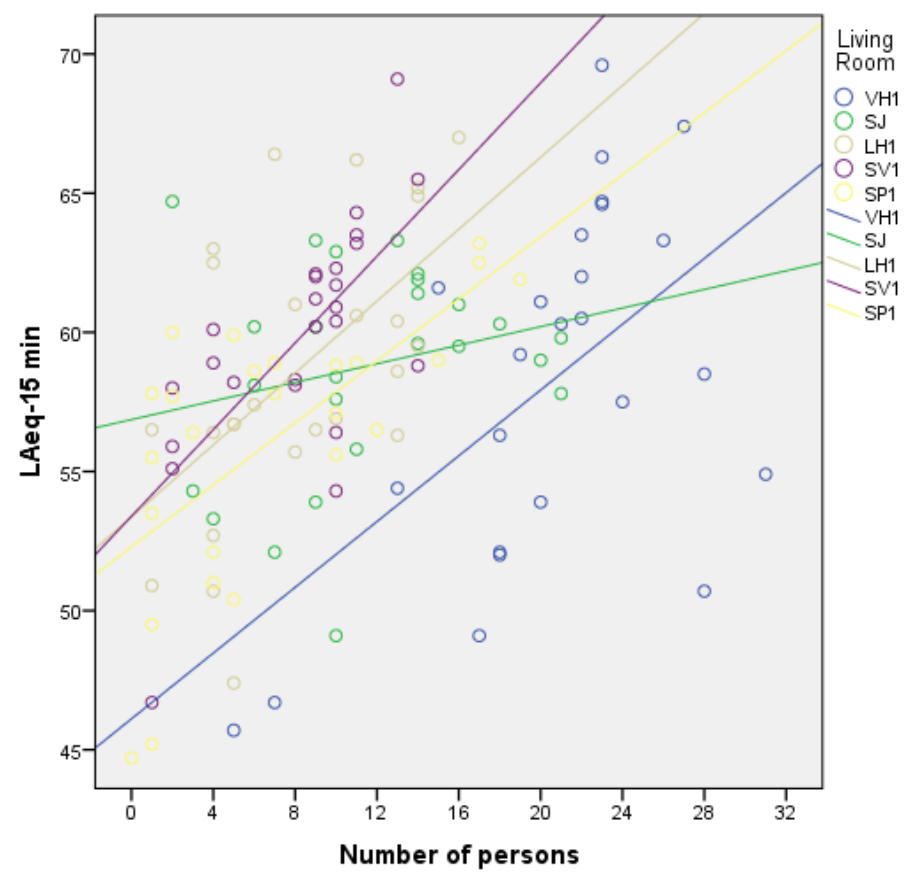

Figure 16. Scatter-plot of the $\mathrm{L}_{\mathrm{A} \text { eq-15min }}$ scores as a function of the number of persons, considering the living rooms separately.

However, when considering all the data together, the linear relationship between sound levels and the number of persons is partially "diluted" and a cubic fit better describes the scatter plot, as shown in Figure 17. This points out that other factors such as the volume and reverberation time of the different living rooms come into play and affect the sound levels, as expected. However, since the slope of the trend lines in Figure 16 is not constant, one can assume that the level of activity of the people in the living rooms is also affecting the sound levels, regardless of the room acoustics conditions.

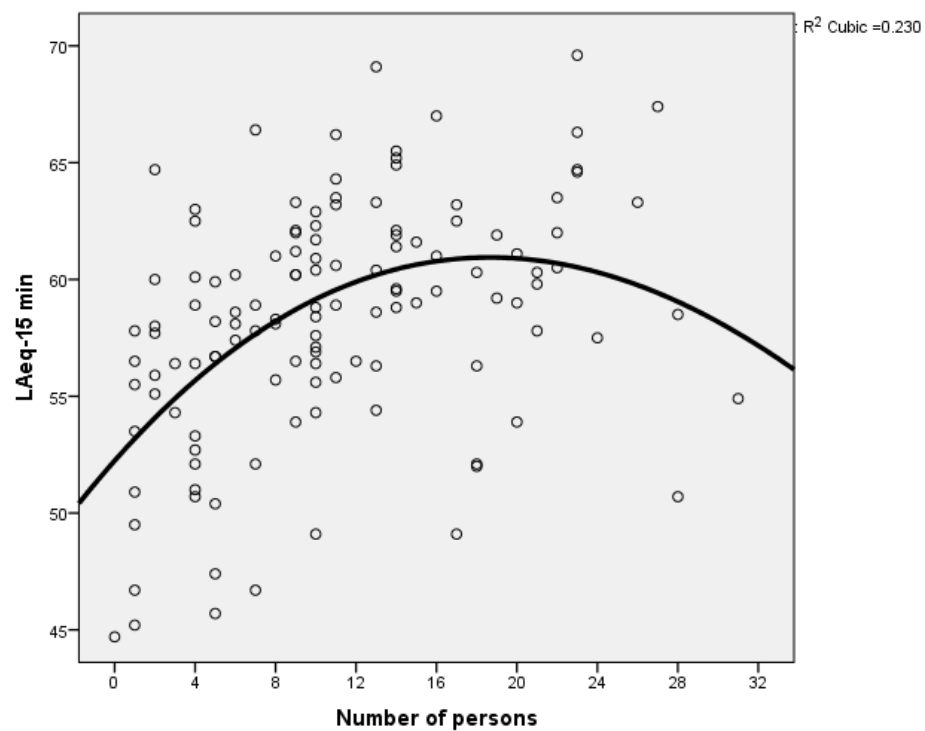

Figure 17. Scatter-plot of the $\mathrm{L}_{\mathrm{Aeq}-15 \mathrm{~min}}$ scores as a function of the number of persons, with cubic fit for all data aggregated. 


\section{Discussion}

\subsection{Differences of Sound Levels and Loudness}

The results reported in Section 3.1 described the differences of sound levels and loudness recorded between different days of the week, different living rooms, and different time slots of the day. To the knowledge of the authors, this was the first study to monitor multiple nursing homes over a one-week period.

The fact that no statistically significant differences were observed in terms of sound levels and loudness in the living rooms between different days of the week was expected, to some extent. It reflects the methodical routine of both staff members and residents in such facilities. Everyday activities (and the sound levels they generate) occur more or less always with the same intensity and over the same periods of time, regardless of the day, in all the nursing homes. This approach seems to be encouraged at a planning and management level in the nursing homes, since the regular temporal variations in the sound environments provide indicators of "audible safety", which is a crucial construct for residents affected by BPSD [1].

When checking for the living room factor, differences of sound levels and loudness did indeed emerge, with some living rooms noisier than others, confirming that each facility has its own "acoustic climate". In general, the noisier living rooms tended to be the medium-sized and slightly over-reverberant ones (see also Table 1 and Figures 8 and 9); however, it was not possible to experimentally control for these factors in particular, since geometries and materials were not modifiable. This suggests that the room acoustics condition is affecting the sound levels, so an acoustic retrofit might actually be a viable strategy to control the sound environment of the living rooms.

The differences in sound levels between different moments of the day were also found to be statistically significant, considering all nursing homes together, with higher levels occurring typically around the breakfast/lunch/dinner times due to higher rates of activity both at the social (e.g., people talking) and functional level (e.g., staff working with kitchenware and serving food). This sound level variability reflects the daily pattern of the living rooms, which is also desirable for its perceptual implications of hints of audible safety [1,19], as mentioned above.

\subsection{Differences of Soundscape Quality}

For the perceptual information (i.e., soundscape data), a smaller dataset was available with respect to sound levels. No statistically significant differences between time slots in terms of any soundscape dimension were observed. Nevertheless, also in this case, differences between the living rooms emerged. As a general trend, the living room SP1 performed worse than others in terms of soundscape quality and appropriateness (i.e., Q1 and Q2), while SJ and SV1 performed slightly better. Nursing homes were shown to have statistically significantly different sound sources profiles, with different degrees of "prominence" of the source types (Q3). When considering the soundscape attributes (Q4), it can be observed that the living rooms LH1 and SP1 were related mostly to monotonous and uneventful soundscapes, while the nursing homes SJ and SV1 performed particularly well in terms of soundscape safety and intimacy, which are dimensions that proved to be extremely important for people with dementia [1,6,7]. For instance, Nagahata et al. [20] showed that elderly people suffering from dementia are able to recollect the sounds that had once occupied very important parts of their lives. However, these sounds in themselves are not unusual sounds in their daily lives. This suggests the importance of soundscape design in daily life.

Taken together, these results suggest that a nursing home's acoustic environment, which is rich and varied in terms of sound sources (like in the cases of VH1 and SJ), might result in better outcomes in terms of overall soundscape quality, while an acoustic environment, which is 'poor' in terms of sound sources prominence and variability (like in the case of SP1), even disregarding their meaning and information content, might not necessarily lead to a good soundscape quality (arguing about the point that "the quieter, the better" should not always be the first choice in soundscape management [12]). 
In soundscape studies, when data collection through questionnaires is included, several participants are usually involved at the same time so that individual responses can be averaged to provide an overall representation of the acoustic environment's perception. However, a different approach was used in this study. A single researcher (F.A.) was trained on the soundscape protocols and performed data collection alone. To some extent, this could imply that the soundscape assessment does not exactly reflect a "general" assessment achieved through the average of multiple participants, and it could potentially introduce a "sample selection bias" [21]. On the other hand, it definitely helps to rule out potential within-group participants effects and provide assessments that are "internally consistent" between contexts and situations. It is worth noting that, within the framework of this study, the (trained) researcher alone assessed the soundscape of the living rooms instead of having the residents appraise these variables themselves, which might not be feasible due to the cognitive condition of the residents. It is not possible to assume that the soundscape assessment of the researcher would be perfectly aligned to the actual experiences of the residents. However, this kind of observational method is increasingly used in soundscape studies in such facilities $[1,6,7]$, with promising results. This methodological approach is also supported by an increasing trend in psychological research to use single-participant experimental designs (for an overview, see ref. [22]), as the potential to provide robust and repeatable empirical findings has been reported across a spectrum of different psychological and behavioural sciences [23]. This circumstance points to the need to further develop alternative methods (e.g., monitoring of physiological parameters) to collect data on the individual responses to the sound environments for people with special needs.

\subsection{Sound Levels and Presence in the Living Rooms}

The results reported in Section 3.3 showed a statistically significant association between the sound levels monitored in the living rooms and the number of people present in those environments during the corresponding time slots. This finding confirms that people are a fundamental sound source in these environments, either because of voices and verbal communication between staff and residents, or because of non-verbal sounds produced by staff members for the functioning of the space.

When considering the data from all living rooms together, it was observed that sound levels usually stopped increasing after a given amount of people in the room was reached. This is possibly due to the fact that residents with BPSD do not interact in the same way, for instance, as an increasing group of people in a social context would do (e.g., it is not uncommon in nursing homes that large people of residents eat in silence), or else, because an increase in the number of people in the room might have reflected a switch from a verbal activity to a "quieter" one (e.g., a large group of residents watching TV all together). However, it was pointed out that the relationship between the number of people and sound levels might also be affected by the room acoustics condition of the single living rooms, which would be in line with previous studies in the literature about the acoustical capacity of dining (or living) spaces [24].

\section{Conclusions}

This research aimed at characterizing the physical sound environment and its perception (i.e., the soundscape) of the living rooms of five nursing homes in Flanders. The main conclusions of this study are:

- Sound levels and loudness levels in the observed living rooms did not vary significantly between days of the week, but they did so between different moments of the day and between different living rooms (and nursing homes).

- Several soundscape quality attributes and the perceived prominence of sound sources types varied significantly between the different living rooms investigated.

- A moderately positive correlation between sound levels and the number of persons in the investigated living rooms was observed. 
Several authors have discussed the issues concerning the stimulation of residents/patients in care facilities through technologies and "active" tools and how this could enhance their physiological and psychological well-being [1,25]. Raising awareness about the potential role of the sound domain in nursing homes is a necessary step towards healthy and stimulating acoustic environments which can promote-and not only permit-better living and working conditions for residents and staff of nursing homes. Active soundscapes, for instance using the residents' sensitivity and preference for specific sounds [1], might be a valuable approach for this to be used by the management of such facilities and should be implemented in their daily practice and organization.

Acknowledgments: The AcustiCare project is supported by the Flemish Agency for Innovation and Entrepreneurship (VLAIO) under the TETRA program for applied research (grant No. HBC.2016.0089). The authors are grateful to the staff members of the nursing homes for their support during data collection, to the residents, and to their relatives.

Author Contributions: Francesco Aletta, Dick Botteldooren, Pieter Thomas, Tara Vander Mynsbrugge, Patricia De Vriendt, Dominique Van de Velde and Paul Devos conceived and designed the study; Francesco Aletta, Paul Devos, Tara Vander Mynsbrugge, and Pieter Thomas collected the data; Francesco Aletta and Paul Devos analyzed the data; all authors wrote the paper.

Conflicts of Interest: The authors declare no conflict of interest. The founding sponsors had no role in the design of the study; in the collection, analyses, or interpretation of data; in the writing of the manuscript, and in the decision to publish the results.

\section{References}

1. Van den Bosch, K.A. Safe and Sound: Soundscape Research in Special Needs Care. Ph.D. Dissertation, University of Groningen, Groningen, The Netherlands, 2015.

2. International Organization for Standardization. ISO 12913-1:2014 Acoustics—Soundscape-Part 1: Definition and Conceptual Framework; ISO: Geneva, Switzerland, 2014.

3. Xiao, J.; Aletta, F. A soundscape approach to exploring design strategies for acoustic comfort in modern public libraries: A case study of the Library of Birmingham. Noise Mapp. 2016, 3, 264-273. [CrossRef]

4. Dokmeci Yorukoglu, P.N.; Kang, J. Analysing Sound Environment and Architectural Characteristics of Libraries through Indoor Soundscape Framework. Arch. Acoust. 2016, 41, 203-212. [CrossRef]

5. Lindborg, P.M.; Friberg, A. Personality Traits Bias the Perceived Quality of Sonic Environments. Appl. Sci. 2016, 6, 405. [CrossRef]

6. Van den Bosch, K.A.; Andringa, T.C.; Başkent, D.; Vlaskamp, C. The Role of Sound in Residential Facilities for People with Profound Intellectual and Multiple Disabilities. J. Policy Pract. Intellect. Disabil. 2016, 13, 61-68. [CrossRef]

7. Van den Bosch, K.A.; Andringa, T.C.; Peterson, W.; Ruijssenaars, W.A.J.J.M.; Vlaskamp, C. A comparison of natural and non-natural soundscapes on people with severe or profound intellectual and multiple disabilities. J. Intellect. Dev. Disabil. 2016, 1-7. [CrossRef]

8. Means, R. Safe as houses? Ageing in place and vulnerable older people in the UK. Soc. Policy Adm. 2007, 41, 65-85. [CrossRef]

9. Vasunilashorn, S.; Steinman, B.A.; Liebig, P.S.; Pynoos, J. Aging in Place: Evolution of a Research Topic Whose Time Has Come. J. Aging Res. 2012. [CrossRef]

10. Dauwe, S.; Van Renterghem, T.; Botteldooren, D.; Dhoedt, B. Multiagent-Based Data Fusion in Environmental Monitoring Networks. Int. J. Distrib. Sens. Netw. 2012. [CrossRef]

11. Domínguez, F.; Dauwe, S.; Cuong, N.; Cariolaro, D.; Touhafi, A.; Dhoedt, B.; Steenhaut, K. Towards an Environmental Measurement Cloud: Delivering Pollution Awareness to the Public. Int. J. Distrib. Sens. Netw. 2014. [CrossRef]

12. Aletta, F.; Kang, J.; Axelsson, Ö. Soundscape descriptors and a conceptual framework for developing predictive soundscape models. Landsc. Urban Plan. 2016, 149, 65-74. [CrossRef]

13. Axelsson, Ö.; Nilsson, M.E.; Berglund, B. A principal components model of soundscape perception. J. Acoust. Soc. Am. 2010, 128, 2836-2846. [CrossRef] [PubMed]

14. Axelsson, Ö. How to measure soundscape quality. In Proceedings of the Euronoise 2015 Conference, Maastricht, The Netherlands, 31 May-3 June 2015. 
15. Aletta, F.; Margaritis, E.; Filipan, K.; Puyana Romero, V.; Axelsson, Ö.; Kang, J. Characterization of the soundscape in Valley Gardens, Brighton, by a soundwalk prior to an urban design intervention. In Proceedings of the Euronoise 2015 Conference, Maastricht, The Netherlands, 31 May-3 June 2015.

16. Aletta, F.; Kang, J. Soundscape approach integrating noise mapping techniques: A case study in Brighton, UK. Noise Mapp. 2015, 2, 1-12. [CrossRef]

17. Fastl, H.; Zwicker, E. Psychoacoustics_Facts and Models; Springer: Berlin, Germany, 1990.

18. Dunn, O.J. Multiple comparisons using rank sums. Technometrics 1964, 6, 241-252. [CrossRef]

19. Campos Costa, I.; Nogueira Carvalho, H.; Fernandes, L. Aging, circadian rhythms and depressive disorders: A review. Am. J. Neurodegener. Dis. 2013, 2, 228-246. [PubMed]

20. Nagahata, K.; Fukushima, T.; Ishibashi, N.; Takahashi, Y.; Moriyama, M. A soundscape study: What kinds of sounds can elderly people affected by dementia recollect? Noise Health 2004, 6, 63-73. [PubMed]

21. Cuddeback, G.; Wilson, E.; Orme, J.G.; Combs-Orme, T. Detecting and Statistically Correcting Sample Selection Bias. J. Soc. Serv. Res. 2004, 30, 19-33. [CrossRef]

22. Morgan, D.L.; Morgan, R.K. Single-participant research design: Bringing science to managed care. Am. Psychol. 2001, 56, 119-127. [CrossRef] [PubMed]

23. Elder, J.H. Single subject experimentation for psychiatric nursing. Arch. Psychiatr. Nurs. 1997, 11, $133-138$. [CrossRef]

24. Rindel, J.H. Verbal communication and noise in eating establishments. Appl. Acoust. 2010, 71, $1156-1161$. [CrossRef]

25. Devos, P.; Min Jou, A.; De Waele, G.; Petrovic, M. Design for personalized mobile health applications for enhanced older people participation. Eur. Geriatr. Med. 2015, 6, 593-597. [CrossRef]

(C) 2017 by the authors. Licensee MDPI, Basel, Switzerland. This article is an open access article distributed under the terms and conditions of the Creative Commons Attribution (CC BY) license (http:/ / creativecommons.org/licenses/by/4.0/). 